\title{
General purpose electronics for real-time processing and encoding of non-MR data in MR acquisitions
}

Pedersen, Jan Ole; Hanson, Christian G.; Xue, Rong; Hanson, Lars G.

Published in:

Concepts in Magnetic Resonance. Part B: Magnetic Resonance Engineering (Online)

Link to article, DOI:

10.1002/cmr.b.21385

Publication date:

2018

Document Version

Publisher's PDF, also known as Version of record

Link back to DTU Orbit

Citation (APA):

Pedersen, J. O., Hanson, C. G., Xue, R., \& Hanson, L. G. (2018). General purpose electronics for real-time processing and encoding of non-MR data in MR acquisitions. Concepts in Magnetic Resonance. Part B: Magnetic Resonance Engineering (Online), [e21385]. https://doi.org/10.1002/cmr.b.21385

\section{General rights}

Copyright and moral rights for the publications made accessible in the public portal are retained by the authors and/or other copyright owners and it is a condition of accessing publications that users recognise and abide by the legal requirements associated with these rights.

- Users may download and print one copy of any publication from the public portal for the purpose of private study or research.

- You may not further distribute the material or use it for any profit-making activity or commercial gain

- You may freely distribute the URL identifying the publication in the public portal 


\title{
General purpose electronics for real-time processing and encoding of non-MR data in MR acquisitions
}

\author{
Jan Ole Pedersen ${ }^{1,2,3}$ (D) | Christian G. Hanson | Rong Xue R,5 $\mid$ Lars G. Hanson $^{1,2}$ (D)
}

\author{
${ }^{1}$ Center for Magnetic Resonance, \\ Department of Electrical Engineering, \\ Technical University of Denmark, \\ Lyngby, Denmark \\ ${ }^{2}$ Danish Research Centre for Magnetic \\ Resonance, Centre for Functional and \\ Diagnostic Imaging and Research, \\ Copenhagen University Hospital, \\ Hvidovre, Denmark \\ ${ }^{3}$ Sino-Danish Center for Education and \\ Research, Aarhus, Denmark \\ ${ }^{4}$ State Key Laboratory of Brain and \\ Cognitive Sciences, Institute of \\ Biophysics, Chinese Academy of \\ Sciences, Beijing, China \\ ${ }^{5}$ Sino-Danish College, University of \\ Chinese Academy of Sciences, Beijing, \\ China \\ Correspondence \\ Lars G. Hanson, Center for Magnetic \\ Resonance, Department of Electrical \\ Engineering, Technical University of \\ Denmark, Lyngby, Denmark. \\ Email: lgh@elektro.dtu.dk
}

\begin{abstract}
Recording of data other than MR signals are often of interest during MRI. We present unique, versatile circuitry developed for sampling and real-time processing of such non-MR signals to facilitate recording of these by an MR scanner. The circuitry is capable of acquiring multiple signals at $200 \mathrm{kHz}$ sampling rate, measure RF power correlates, perform fast and flexible signal processing, and transmitting both amplitude and frequency modulated RF signals receivable by MR scanners. As an example of use, an electronic point-source signal is generated by the circuitry, and transmitted wirelessly to the receive coil of an MR scanner during an echo planar imaging sequence.
\end{abstract}

\section{1 | INTRODUCTION}

Signals other than the magnetic resonance (MR) signal are often of interest during magnetic resonance imaging (MRI). These signals of interest (SOIs) can, for example, contain biomedical information such as electroencephalography $(E E G)^{1,2}$ or be used for scanner monitoring and characterization. An example of the latter is gradient field measurements, which facilitate determining the exact position of each MR sample in $k$-space. ${ }^{3-5}$ When acquiring such SOIs, care must be taken to avoid interference with MR measurements, and avoid artefacts caused by scanner subsystems, typically dominated by radio frequency (RF) transmission and gradient switching. Depending on the SOI in question, different strategies have been used. For audio recordings, subtracting a noise-only signal acquired separately has been shown to be a viable strategy. ${ }^{6}$ For EEG recording, a typical approach is to minimize artefacts, by, eg, twisting bipolar electrodes, and filter out remaining artefacts in post-processing. ${ }^{1,7} \mathrm{~A}$ third approach is to only sample the SOIs in periods where the interfering sources are not active, such as during gradient plateaus, coinciding with MR signal acquisition. ${ }^{8}$ While this makes filtering of the SOI to remove artefacts a trivial, or even unnecessary task, it requires high temporal precision and synchronization with the MR scanner, which is non-trivial to obtain.

A relatively simple strategy to obtain the necessary synchronization is acquisition of the SOIs by the MR scanner, which we previously presented hardware developed to facilitate. ${ }^{9,10}$ The SOIs were amplitude modulated at carrier frequencies close to the demodulation frequency of the scanner, and wirelessly transmitted to the MR receive coil. The amplitude modulation, which entailed unattenuated transfer through the scanner's receiver filters, was demodulated in the 
scanner's receive chain, enabling extraction of the SOIs from the raw data of the scan. Non-interfering concurrent SOI and MRI acquisition was possible by exploiting that readout oversampling followed by bandwidth reduction is normally done in MR image reconstruction, allowing for encoding the amplitude modulated SOIs in the discarded frequency range. For traditional Cartesian MRI sampling, the amplitude modulated SOIs thus appeared as stripes orthogonal to the frequency encoding direction outside the requested FOV, if reconstruction was performed without bandwidth reduction. For echo planar imaging (EPI), where the MR signal and modulated SOIs were most easily separated by individual Fourier transformation of each sampled $k$-space line, the sampling rate of the SOIs effectively became the reciprocal of the echo-spacing, thus typically in the $\mathrm{kHz}$ range. This was shown to be sufficient for concurrent acquisition of biomedical signals, including electrooculography, electrocardiography, and EEG. ${ }^{10}$ Use of the oversampled bandwidth has recently also been suggested for respiratory monitoring by acquisition of thermal noise, ${ }^{11}$ or by determining the coupling of an antenna transmitting a single-frequency RF signal to the scanner's receive coil. ${ }^{12}$ Since readout oversampling is default for MRI, use of the discarded bandwidth is generally possible, also for non-Cartesian k-space sampling and parallel imaging.

A subset of SOIs cannot be directly sampled using the previously presented hardware. These include SOIs with higher frequency content than the available sampling bandwidth, SOIs having complicated dependencies (example below), and/or SOIs, where sampling gaps between scanner readouts are not acceptable. This subset of SOIs can typically still be sampled using an MR scanner, if appropriate signal processing is done before transmission to the scanner. The required pre-processing is dependent on the signal in question, and a catch-all solution is therefore not feasible. One example is electronic phantoms, where the signal from a chosen transversal magnetization density is determined by integrating the Bloch equations and emulated electronically. ${ }^{13}$ As the accumulated phase of an MR signal at a given time point is dependent on the magnetic field history, particularly since the most recent excitation pulse, knowledge of both the scanner-generated RF field $\left(B_{1}(t)\right)$ and the gradient field must be incorporated into a single SOI to perform such emulation.

To facilitate MR scanner sampling of a broader category of SOIs, we present tailored circuitry capable of sampling up to three signals simultaneously with acquiring a correlate of the RF power transmitted by MR scanners. Real-time flexible processing of the incoming signals is performed by a Field Programmable Gate Array (FPGA), which also controls the phase, frequency and amplitude of a circuitry-generated signal that is receivable by MR scanners.

A photograph and schematics of the open-source circuitry are shown in Figure 1, and a further description and characterization is given in Section 2. The full circuit board layout is additionally provided at http://drcmr.dk/Magstripe Encoding. While FPGA-based electronics previously have been developed for processing of MR signals, also outside of scanners, ${ }^{14}$ this is to our knowledge the first developed for transmitting scanner-receivable signals, which offers several advantages. The presented circuitry is the first generation and limited optimization has been carried out. Yet it shows considerable potential.

As a putative use case of the circuitry, an MR pointsource is emulated electronically from measured $B_{1}(t)$ and gradient field activity during an MRI sequence. Emulation of such a signal is highly demanding in terms of bandwidth, signal-to-noise ratio and timing, and thus demonstrates the performance and limitations of the circuitry well. Even when limited to a point-source, electronic phantoms have multiple potential uses. Examples include motion sensing, sequence optimization, quality assurance, and signal quantification. The electronically generated signal recorded by the MR scanner is compared to simulations, and the signal obtained from imaging of a physical point-like source.

\section{2 | MATERIALS}

For fast and flexible signal processing, the presented circuitry features an FPGA (Max 10, Altera Corporation San Jose, California, USA. Clock frequency: $40 \mathrm{MHz}$ ), which receives data from three 16-bit ADCs for low frequency signal sampling (denoted LF-Rx, sampling rate: $200 \mathrm{kHz}$, maximum signal peak-peak amplitude $\left.\left(U_{\mathrm{pp}}\right)=2 \mathrm{~V}\right)$. Before reaching the ADCs, an incoming signal is passed through a programmable-gain amplifier (PGA) with possible amplification between $1 \times$ and $200 \times$, and a lowpass antialiasing filter (22 kHz cutoff frequency). The FPGA receives a non-linear power correlate from a fourth ADC that detects 50$1000 \mathrm{MHz}$ signals in a $-25 \mathrm{dBm}$ to $14 \mathrm{dBm}$ range (RF-Rx).

In our previous work, ${ }^{10}$ transmission of a SOI to the MR scanner was wireless, which for concurrent MR acquisition was viable, provided the modulated SOIs were detectable and had no spectral overlap with the MRI signal. This restricted the bandwidth available for SOI sampling. By using a wired connection to a single receive channel of a scanner allocated to the purpose, the full channel bandwidth is available for sampling of a SOI with no risk of signal contamination. Depending on the MR scanner and receive coil in question, this may be at the cost of reduced signal-to-noise ratio (SNR) of the MR signal, as the allocated receive channel is not available for MR reception. For the receive coils, where this method has been tested, shunting the output of the preamplifier of the unconnected coil element with the impedance of the receive channel 

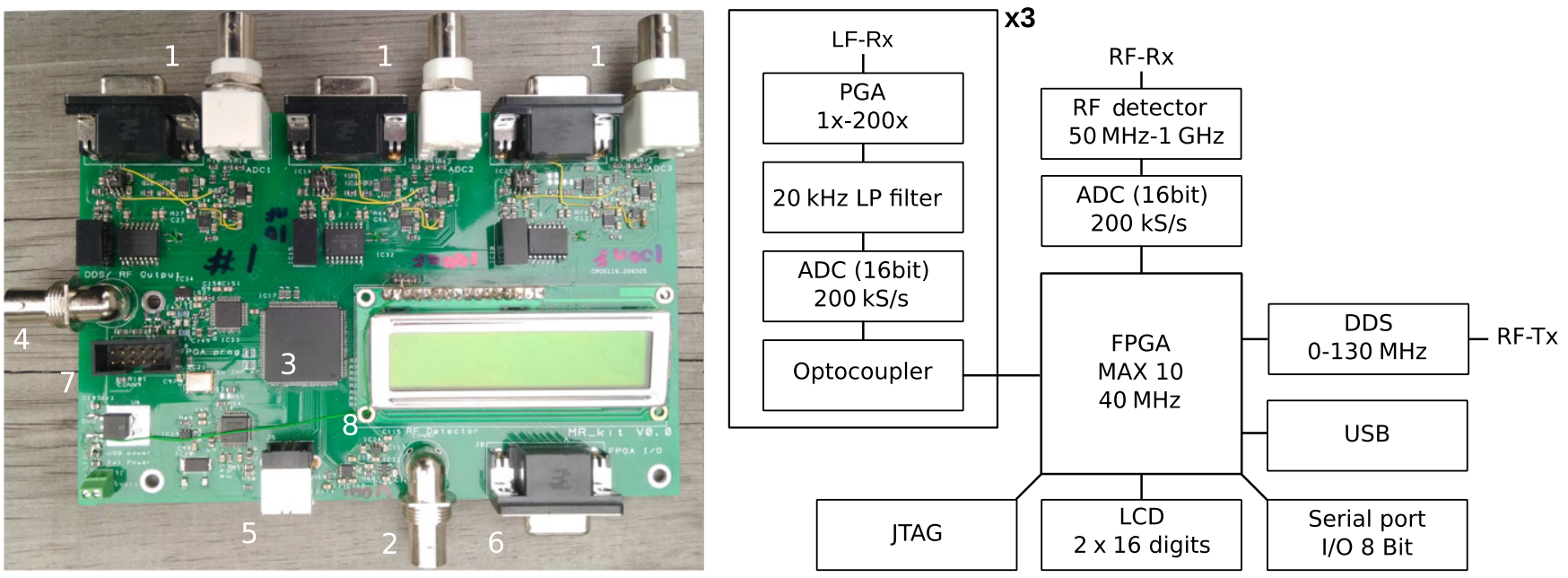

F I G URE 1 Photograph (left) and schematics (right) of the circuitry developed for acquisition of non-MR data by MR scanners. Numbering in parenthesis corresponds to numbering given in the photograph. Low-frequency signal sources are connected by either BNC or a Dsubminiature port, which also features a voltage supply for external equipment (1). RF-signal sources are connected by BNC (2). After digitization, received signals are transmitted to a FPGA (3) which also controls the output (4) of a DDS generating RF-signals receivable by MR scanners. Two-way communication with the FPGA is possible by USB (5) or a serial interface (6), and it can be flashed by a JTAG interface (7). The circuitry also features an LCD (8) used for showing the status of the circuitry

ensured unchanged behavior of the coil element. A wired connection poses a potential safety risk, as current flow to the scanner or ground is possible. To avoid this, signals from LF-Rx to the FPGA are passed through an optocoupler, providing galvanic isolation.

For transmission of a SOI to the scanner, the FPGA controls the output of a direct digital synthesizer (DDS) subcircuitry, yielding phase, amplitude, and frequency control of an output signal (RF-Tx) of up to $130 \mathrm{MHz}$ frequency and amplitude up to $U_{\mathrm{pp}}=100 \mathrm{mV}$. Two-way communication for hardware control and signal visualization on a computer is possible through a Universal Serial Bus (USB) connection and an 8-bit serial port. The use of the available bandwidth is fully customizable, as it is controlled by the FPGA. For the work presented here, the USB connection was used for transmission of data packages containing address, time stamp, and six 16-bit samples at $300 \mathrm{kHz}$ for monitoring measured and FPGA-internal signals. A Joint Test Action Group (JTAG) interface makes flashing of the FPGA possible, and a $2 \times 16$ digit liquid crystal display (LCD) controlled by the FPGA is featured for simple FPGA feedback. The board can either be powered through USB or by a $5 \mathrm{~V}$ DC supply, eg, a non-magnetic rechargeable battery, facilitating positioning of the circuitry in the fringe field of an MR scanner.

\section{3 | METHODS}

To determine the noise properties of the output of the circuitry, a sinusoidal signal $\left(U_{\mathrm{pp}}=1 \mathrm{~V}\right.$, frequency $f=1000 \mathrm{~Hz}$ ) was sampled (LF-Rx, PGA gain: $1 \times$ ) and modulated by example carrier frequencies $63.87 \mathrm{MHz}$
(Larmor frequency of ${ }^{1} \mathrm{H}$ at $\left.1.5 \mathrm{~T}\right), 127.74 \mathrm{MHz}\left({ }^{1} \mathrm{H}\right.$ at $3 \mathrm{~T}$ ), $32.13 \mathrm{MHz}\left({ }^{13} \mathrm{C}\right.$ at $\left.3 \mathrm{~T}\right)$ and $74.97 \mathrm{MHz}\left({ }^{13} \mathrm{C}\right.$ at $\left.7 \mathrm{~T}\right)$.

The feasibility of extracting signals that are amplitude modulated by the circuitry and received by an MR scanner was also tested. Input waveforms ( 1 second burst, triangular amplitude, $U_{\mathrm{pp}}=1 \mathrm{~V}, f=1 \mathrm{~Hz}, 10 \mathrm{~Hz}, 100 \mathrm{~Hz}$ and $1000 \mathrm{~Hz}$ ) were generated by a waveform generator (Keysight 33500B, Santa Rosa, CA, USA) and amplitude modulated with the ${ }^{1} \mathrm{H}$ demodulation frequency of a $3 \mathrm{~T}$ Philips Achieva MRI scanner $(\approx 127 \mathrm{MHz})$ before being wirelessly transmitted during a pulse-acquire sequence (bandwidth: $8000 \mathrm{~Hz}$, readout time: 2.04 second). The waveforms were transmitted 0.5 second after the start of the scanner readout. The waveforms were extracted from the acquired MR data and compared to the input waveform, simultaneously acquired by an oscilloscope (Agilent Technologies, Santa Clara, CA, USA DSOX3024A, voltage resolution: $5 \mathrm{mV}$ ).

A rudimentary electronic phantom was generated by measuring the local temporal variation of the scanner's gradient field, $B_{\mathrm{G}}(\mathbf{r}, t)$, during an EPI sequence, and modulating the frequency of an emulated, exponentially decaying signal, $S_{\mathrm{e}}(t)$, transmitted to the MR scanner correspondingly. For comparison, a point-source signal was also obtained from simulations, $S_{\mathrm{s}}(t)$, and imaging of a pointlike physical phantom, $S_{\mathrm{p}}(t)$. The signals $S_{\mathrm{s}}(t)$ and $S_{\mathrm{e}}(t)$ were generated to have exponential decay matching the $T_{2}$ decay of the physical phantom, being excited by a homogeneous $B_{1}(t)$ under steady-state incoherent conditions. ${ }^{15}$

The physical phantom $(6 \mathrm{~cm}$ water filled $\varnothing 1.5 \mathrm{~mm}$ PVC tubing) was placed at target position $\mathbf{r}$ with its axis perpendicular to the image plane and oriented along the scanner's static field, $\mathbf{B}_{0}=\hat{z} \cdot B_{0}$, where $\hat{z}$ is a unit vector along the 
$z$-axis chosen along the scanner bore. A single-slice, singleshot EPI sequence was used (TE/TR: $28 \mathrm{~ms} / 4000 \mathrm{~ms}$, dwell-time: $1.42 \mu \mathrm{s}$, echo-spacing: $394 \mu \mathrm{s}, 90^{\circ}$ tip angle, voxel size: $4 \times 4 \mathrm{~mm}$, slice thickness: $4 \mathrm{~mm}$, FOV: $500 \mathrm{~mm} \times 500 \mathrm{~mm}$, readout direction oversampling factor: $2)$. To improve the obtained SNR of $S_{\mathrm{p}}(t)$, the evaluated signal was obtained from averaging across 128 repeated acquisitions. The large FOV was chosen to provide large voxels, thus making the physical phantom a good pointsource approximation. An EPI sequence was used, as the reconstructed images effectively feature two frequency encoding axes (readout direction and blip direction), as reflected in the bandwidth per pixel (bpp) that differs accordingly between the two axis $\left(b_{p p} \mathrm{RO}=3039 \mathrm{~Hz}\right.$, bpp $_{\text {blip }}=20.35 \mathrm{~Hz}$ ). Additionally, large gradient field strengths and slew rates are applied, making EPI a particularly challenging example for the circuitry, as the dynamic range of the induced signal is considerably larger than for other sequences. For the acquisition of the emulated signal, $S_{\mathrm{e}}^{m}(t)$, no averaging was performed (50 repeats, individually identified by superscript $m$ ). The scanner's data acquisition was delayed to account for a known signal delay through the circuitry $(15 \mu \mathrm{s})$. As time for $T_{1}$ relaxation was not needed for the emulation, TR was here reduced to $100 \mathrm{~ms}$. To determine $B_{G}(\mathbf{r}, t)$ a gradient pick-up coil (Ø3 cm, 20 windings) was centered at position $\mathbf{r}$, with the normal vector of its circular cross section oriented perpendicular to $\mathbf{B}_{0}$. This yielded an electromotive force,

$$
\varepsilon_{\mathbf{r}}(t)=-\frac{d \Phi_{G}}{d t}=\frac{1}{b} \cdot \frac{d B_{G}(\mathbf{r}, t)}{d t}
$$

where $\Phi_{G}$ denotes the magnetic flux through the pick-up coil and $b$ denotes a geometry factor of the pick-up coil. The product of $\mathrm{B}_{G}(\mathbf{r}, \mathrm{t})$ and the emulated gyromagnetic ratio, $\gamma$, yield the gradient-induced precession frequency offset, which was determined as

$$
\gamma B_{G}(\mathbf{r}, t)=f_{G}(\mathbf{r}, t)=\gamma b \int_{0}^{t} \varepsilon_{\mathbf{r}}\left(t^{\prime}\right) d t^{\prime}+c
$$

where $t=0$ denotes the isodelay point ${ }^{16}$ of the latest excitation pulse and $c$ accounts for possible gradients applied at the isodelay point, eg, a slice selection gradient. The expression holds as long as the gradient field is linearly varying over the cross section of the pick-up coil, which was assumed a valid approximation as the pick-up coil was relatively small and positioned inside the normal imaging volume of the MR scanner. Due to the long TR for imaging of the physical phantom compared to $T_{1}$ of water, only the primary FID following excitation was assumed to contribute to the signal. Echo pathways were thus ignored.

The solution to the integral in Equation (2) was approximated as a running sum of $\varepsilon_{\mathbf{r}}(t)$ measured by LF-Rx. To challenge the circuitry, the position $\mathbf{r}$ (approximately $7.5 \mathrm{~cm} / 15 \mathrm{~cm}$ offcenter in readout/blip-directions) was chosen so that during the dwell-time of LF-Rx, $\Delta t=5 \mu \mathrm{s}$, the maximum nominal change of $f_{G}(\mathbf{r}, t)(2.54 \mathrm{kHz})$ was comparable to the frequency resolution in the readout direction of the EPI sequence. The circuitry was thus expected to correctly position the point-source in the readout-direction, but with limited precision in the higher resolved blip-direction.

The $T_{2}$ of the physical phantom was determined using multiple pulse-acquire sequences with varying echo time, and was used in the emulation $\left(T_{2}=1.4\right.$ second). Since this was considerably longer than the time between signal amplitude updates (for simplicity chosen equal to $\Delta t$ ), the exponential decay of the amplitude of $S_{\mathrm{e}}(t)$ was wellapproximated using the Euler method. At $t=0, M_{\perp}$ was set to a preset value, corresponding to the size of the longitudinal magnetization in equilibrium, thus emulating perfect $90^{\circ}$ unsaturated excitation. The calculated signal was frequency modulated to the demodulation frequency of the MR scanner by the DDS subcircuitry to emulate precession around $\mathbf{B}_{0}$. The generated MR-like signal was transmitted wirelessly to the receive coil of the scanner through a quarter wavelength antenna connected to RF-Tx. An identical antenna was connected to RF-Rx for monitoring $B_{1}(t)$. As all RF pulses in the sequence were excitation pulses with maximum amplitude at the isodelay, $t=0$ was simply determined by the RF-Rx signal exceeding a threshold. The pick-up coil was observed to also be partially sensitive to $B_{1}(t)$, causing gradient waveforms concurrent with $B_{1}(t)$ to be noisily determined. This caused incomplete cancellation of the slice selection gradient and the following refocusing gradient, and therefore an offset in the frequency of $S_{\mathrm{e}}(t)$. To avoid this, both gradient waveforms were turned off, thus emulating a perfect slice selection, and resulting in $c=0$. The PGA was set to $2 \times$ amplification, and the constant $b$ was chosen so that the emulated point-source was positioned at $\mathbf{r}$ in a prior calibration scan. The value 1 was subtracted from $\varepsilon_{\mathbf{r}}(t)$ in a fraction of the time steps to account for a sub-bit bias of LFRx. The frequency of this subtraction was chosen so that measured positive and negative gradients were balanced for the EPI sequence. For both acquisition of $S_{\mathrm{e}}(t)$ and $S_{\mathrm{p}}(t)$, only data acquired from a single receive element of a 32 channel head coil was used in the following analysis.

The signal $S_{\mathrm{s}}(t)$ was obtained by solving the Bloch equations. This simulation was based on gradient waveforms predicted by the MR scanner, which are obtained by convolving the nominal gradient waveforms with a kernel determined by the vendor during calibration of the MR scanner. The kernel accounts for most of the gradient hardware imperfections, such as slew rate limitations, but does not include, eg, spatial and temperature dependencies. ${ }^{17}$

The ability of the circuitry to correctly emulate the temporal phase evolution of a point-source signal was 
investigated by comparing the phase evolution from individual repetitions of $S_{\mathrm{e}}(t)$ to $S_{\mathrm{p}}(t)$ and $S_{\mathrm{s}}(t)$. Following this, the ability of the circuitry to yield a point-source in a reconstructed image was investigated. The ramp-sampled $S_{\mathrm{e}}(t)$ and $S_{\mathrm{p}}(t)$ were reconstructed by non-uniform Fourier Transformation ${ }^{18}$ using a $k$-space trajectory based on the scanner-predicted gradient waveforms. An effective delayed play-out of the gradient waveforms results in a Nyquist ghost, that was not corrected for, and a stronger ghost than typically observed in EPI images is therefore expected. The reconstructed images were normalized to have equal maximum amplitude. Based on the reconstructed images of $S_{\mathrm{e}}(t)$, the position of the emulated point-source was determined as the highest intensity voxel, and compared to the position of the physical phantom, determined from reconstruction of $S_{\mathrm{p}}(t)$. The circuitry was positioned in the operator room for all presented experiments.

\section{4 | RESULTS}

The spectra of the amplitude modulated sinusoids generated by the circuitry are depicted in Figure 2 in a -50 to $50 \mathrm{kHz}$ range around the carrier frequencies. The individual signals were present at three frequencies, consistent with the expected spectrum of an amplitude modulated single frequency signal. A similar noise floor $-80 \mathrm{~dB}$ below the signal was observed for all four frequency ranges. For $74.97 \mathrm{MHz}$, additional noise was observed around $-25 \mathrm{kHz}$. The cause of this noise was not investigated further, and the signal power was still significantly higher $(30 \mathrm{~dB})$.

The triangular waveforms acquired by an oscilloscope and the MR scanner after amplitude modulation, were found to be almost identical, as seen in Figure 3 (top) where both are depicted for one example of the tested waveforms $(f=10 \mathrm{~Hz})$. Their difference, Figure 3 (mid), was anticorrelated with the test signals, indicating a slight attenuation of the test signals sampled by the MR scanner. Rescaling the scanner-acquired waveform by a factor 1.012 reduced the difference to a level comparable to the variation seen before transmission of the burst signal, depicted in Figure 3 (bottom). After rescaling, the signal difference had a standard deviation of $3.2 \mathrm{mV}$, corresponding to $0.64 \%$ of the peak amplitude of the waveform generator output. For comparison, an average standard deviation of $2.7 \mathrm{mV}$, or $0.54 \%$ of the peak amplitude, was found across 50 repetitions of the scanner acquisitions. Due to the limited resolution of the oscilloscope, it was not determined, whether the deviation between repetitions was caused by the circuitry or the waveform generator.

The phase evolution during three repeated acquisitions of $S_{\mathrm{e}}(t)$ is depicted in Figure 4 (left). The difference in phase, $\Delta \varphi$, between $S_{\mathrm{e}}(t)$ and $S_{\mathrm{s}}(t)$ is depicted in Figure 4 (top right) for the full duration of the repetitions, and Figure 4 (bottom right) for the first 10 readout periods of the same repetitions. Of the 50 repetitions of $S_{\mathrm{e}}(t)$, the three depicted showed the largest negative $\Delta \varphi\left(S_{\mathrm{e}}^{27}(t)\right.$, red), largest positive $\Delta \varphi\left(S_{\mathrm{e}}^{29}\right.$, green), and smallest final $\Delta \varphi\left(S_{\mathrm{e}}^{17}(t)\right.$, blue), respectively. The repetitions $S_{\mathrm{e}}^{27}(t)$ and $S_{\mathrm{e}}^{29}(t)$
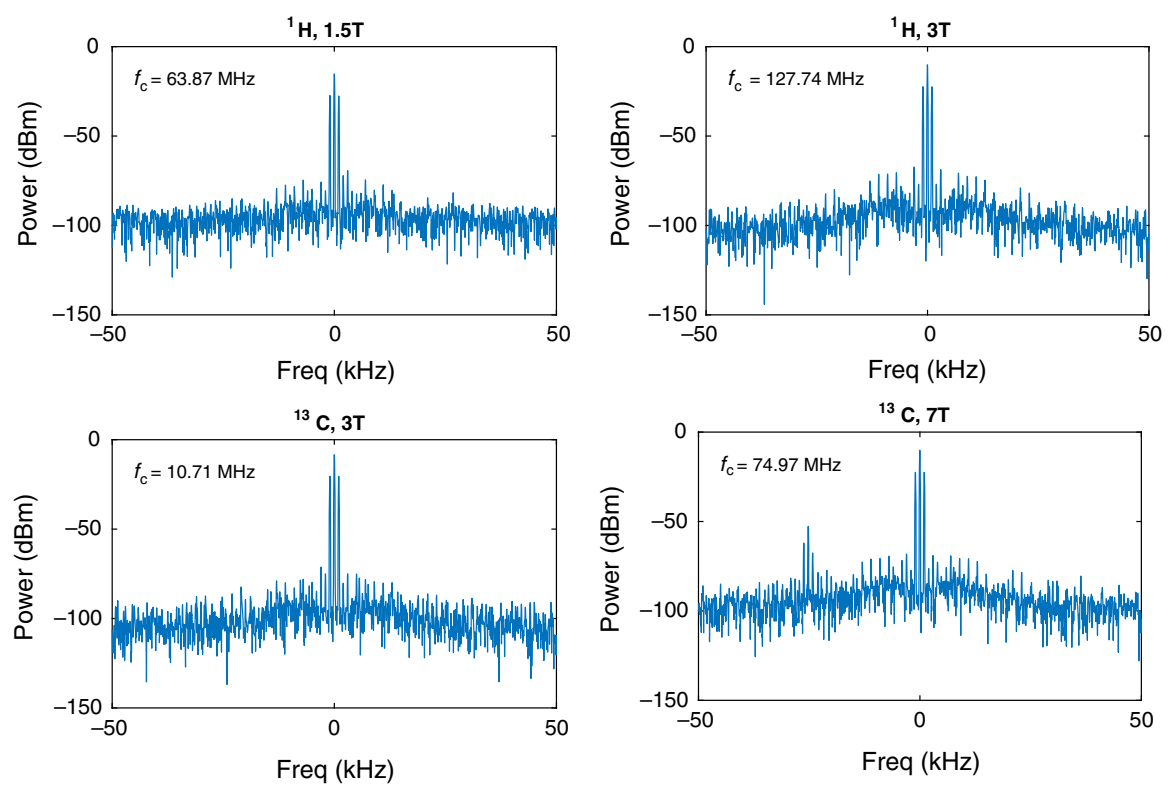

FIGURE 2 Measured output power of amplitude modulated sinusoidal signals $\left(U_{\mathrm{pp}}=1 \mathrm{~V}, f=1000 \mathrm{~Hz}\right)$ shown as a function of frequency offset from four example carrier frequencies $f_{\mathrm{c}}=63.87,127.74,10.71$, and $74.97 \mathrm{MHz}$. Similar power levels are seen at the carrier $(-15$ to $-8 \mathrm{dBm})$ and sideband frequencies $(-27$ to $-20 \mathrm{dBm})$. Besides additional noise around $-25 \mathrm{kHz}$ from $74.97 \mathrm{MHz}$, a common noise floor $(-95 \mathrm{dBm})$ is seen for all four frequency intervals 

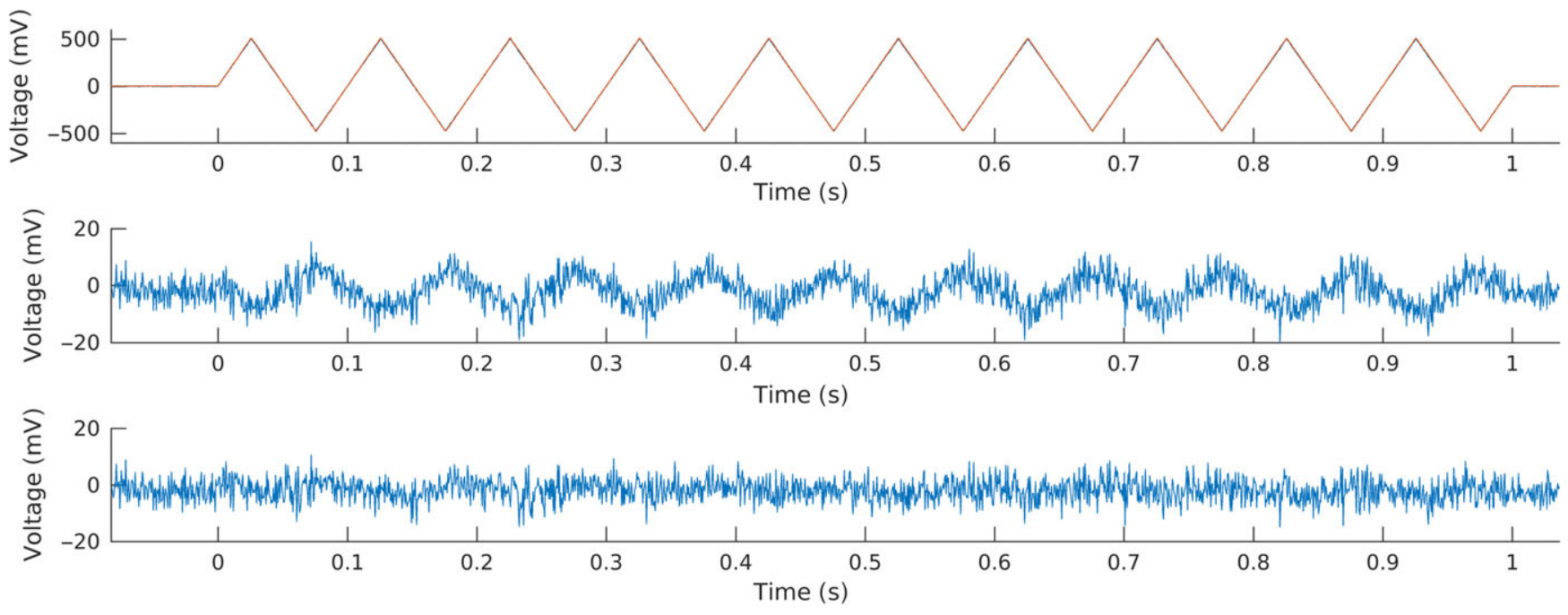

FIGURE 3 Top: A $10 \mathrm{~Hz}$ test waveform extracted from measurements performed with a pulse-acquire MR sequence (red curve) and an oscilloscope (blue curve, mostly hidden under red curve), where time $t=0$ corresponds to start of the test signal play-out. Mid: Difference between the test signals acquired by the scanner and the oscilloscope. The mean absolute difference corresponds to $0.83 \%$ of the maximum signal amplitude. Bottom: Difference between rescaled scanner-acquired and oscilloscope-acquired test signal. The mean absolute difference corresponds to $0.57 \%$ of the maximum signal amplitude
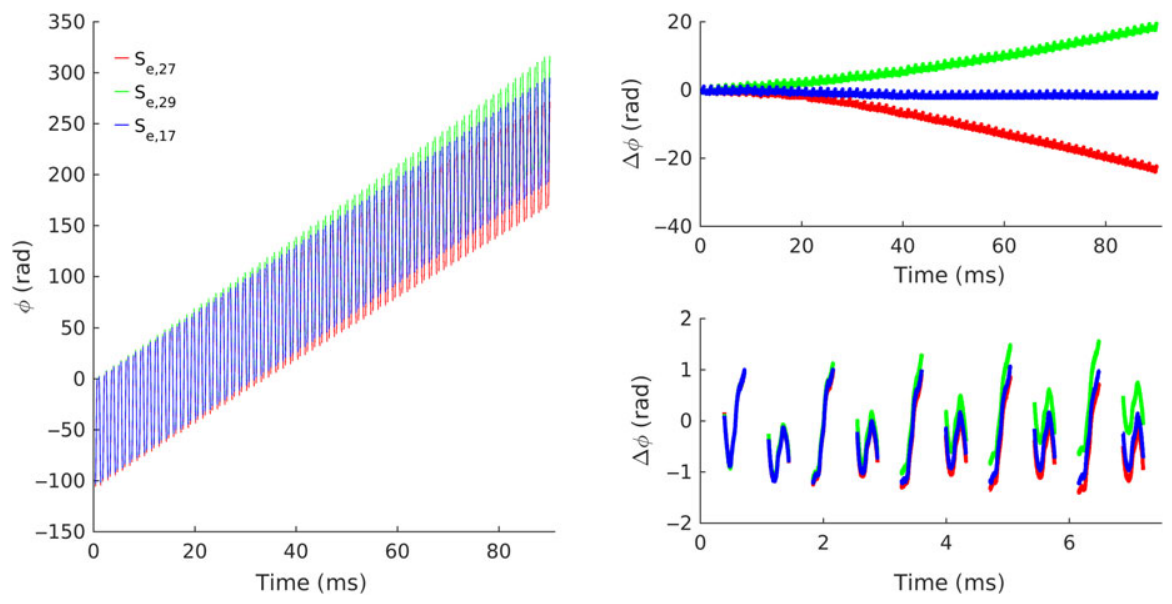

FIGURE 4 Left: Phase evolution of three repetitions of $S_{\mathrm{e}}(t)$. Right: Difference between the phase evolution of the three repetitions of $S_{\mathrm{e}}(t)$ and $S_{\mathrm{s}}(t)$ for the entire repetition (top) and for the first 10 readout periods (bottom)

showed similar magnitude of $\Delta \varphi$, corresponding to $\varepsilon(t)$ being determined with a constant offset of $95 \mu \mathrm{V}$ and $-95 \mu \mathrm{V}$ (before PGA amplification), or \pm 6.1 times the smallest voltage increment $(\Delta v)$ of LF-Rx after $2 \times$ PGA amplification. For comparison, in periods without gradient waveforms, but with the gradient amplifier turned on and idle, $\varepsilon(t)$ was white noise with a standard deviation of $519 \mu \mathrm{V}(34 \cdot \Delta v)$. On the shorter time scale of a single readout period, all three repetitions show almost identical phase evolution. Similar behavior was observed for non-depicted repetitions. The final phase deviation showed a normal distribution corresponding to a standard deviation of $208 \mathrm{rad}$ $\mathrm{s}^{-1}$, or a standard deviation in the measured magnetic field of $781 \mathrm{nT}$.

The phase evolutions of $S_{\mathrm{e}}^{17}(t)$ and the physical phantom signal, $S_{\mathrm{p}}(t)$, are compared in Figure 5, where the phase of $S_{\mathrm{s}}(t) / S_{\mathrm{e}}^{17}(t)$ (blue) and $S_{\mathrm{s}}(t) / S_{\mathrm{p}}(t)$ (black) are depicted. The phase of $S_{\mathrm{p}}(t)$ appeared to have considerable high-frequency content that increased in amplitude over time, and decreased with inclusion of more averages (data not shown), which is consistent with this being thermal noise. Similar behavior was not seen in the phase of any repetition of $S_{\mathrm{e}}(t)$, supporting the thermal noise hypothesis. Additionally the signal deviated from that expected from a true point-source, as the phase of $S_{\mathrm{p}}(t)$ showed an unexpected slow oscillation, and the signal strength decayed with a relaxation rate, $T_{2}^{*}$, faster than its determined $T_{2}$.

Overall, $S_{\mathrm{p}}(t)$ and $S_{\mathrm{e}}^{17}(t)$ showed a similar phase deviation from $S_{\mathrm{s}}(t)$, indicating a significant difference between the actual and nominal gradients. After correction for possible differences in the initial phase of the signals, the rootmean-square (RMS) error between $S_{\mathrm{p}}(t)$ and $S_{\mathrm{s}}(t)$ was $0.55 \mathrm{rad}$, whereas between $S_{\mathrm{s}}(t)$ and $S_{\mathrm{e}}^{17}(t)$ this was found to be $0.48 \mathrm{rad}$. For the worst emulated case, $S_{\mathrm{e}}^{27}(t)$, the 

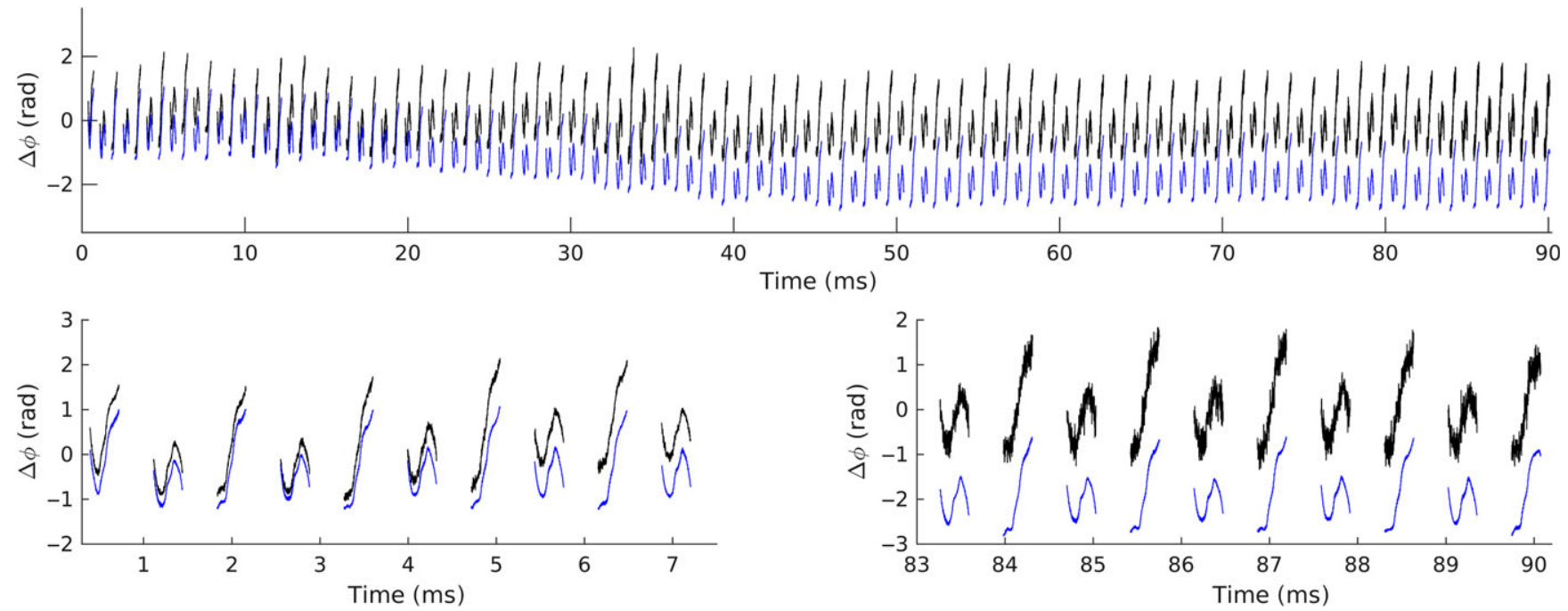

FIGURE 5 Temporal phase evolution of $S_{\mathrm{s}}(t) / S_{\mathrm{e}}^{17}(t)$ (blue) and $S_{\mathrm{s}}(t) / S_{\mathrm{p}}(t)$ (black) for the entire $k$-space traversal (top), the first 10 readout periods (bottom left) and the last 10 readout periods (bottom right)

RMS error was found to be $46 \mathrm{rad}$. To estimate the error if $S_{\mathrm{e}}(t)$ is generated from only integrating $\varepsilon(t)$ over each readout period instead of overall repetitions, the RMS error was also determined after mean-subtraction of the phase for each individual readout-period of $S_{\mathrm{e}}(t)$ and $S_{\mathrm{p}}(t)$. The RMS error for all $S_{\mathrm{e}}(t)$ was then found to be between 0.16$0.18 \mathrm{rad}$.

The reconstructed images of $S_{\mathrm{p}}(t)$ (left) and of two repetitions of $S_{\mathrm{e}}(t)$ are depicted in Figure 6 with linear and logarithmic amplitude scaling $\left(S_{\mathrm{e}}^{17}(t)\right.$, center and $S_{\mathrm{e}}^{27}(t)$, right). The position of the point-source was correctly emulated in the readout direction (left-right) for all 50 repetitions of $S_{\mathrm{e}}(t)$. In the blip-direction, the deviation in the phase evolution lead to variation of the phantom position between +5 and -3 pixels around the target position $(-102$ to $61 \mathrm{~Hz})$. The cumulative nature of the error corresponds to an effective temporal non-linear blip-gradient variation, causing the point-source signal to be smeared over multiple pixels in the blip-direction, particularly visible for $S_{\mathrm{e}}^{27}(t)$.

The reconstructed magnitude images of all repetitions of $S_{\mathrm{e}}(t)$ showed non-Gaussian noise, though only visible on logarithmically scaled images. The relative noise level was lower than that was observed in the reconstructed image of $S_{\mathrm{p}}(t)$. This was evident when Gaussian noise was added to the individual repetitions of $S_{\mathrm{e}}(t)$ to yield the same SNR as the physical point-source image, and the Gaussian noise dominated the structured noise (not shown). A half FOV ghost was also observed for all reconstruction of $S_{\mathrm{e}}(t)$ and $S_{\mathrm{p}}(t)$ with similar relative amplitude (11\%-13\%). This ghost was attributed to the reconstruction being based on the $k$ space trajectory expected by the scanner, which from Figure 5 was concluded to deviate from the actual $k$-space trajectory. Besides the half FOV ghost, the reconstructed image of $S_{\mathrm{p}}(t)$ showed additional aliases around $-80 \mathrm{~Hz}$ and
$250 \mathrm{~Hz}$, as a result of the slow oscillations visible in the phase evolution of $S_{\mathrm{p}}(t)$ observed in Figure 5.

\section{5 | DISCUSSION}

In this article, we presented unique circuitry developed to facilitate sampling of non-MR signals by an MR scanner. Signals transmitted by the circuitry showed high SNR, and acquisition by an MR scanner introduced only minimal distortions, that were easily accounted for. By combining an inductive gradient field measure and $B_{1}(t)$ power measurements, the circuitry was shown capable of emulating a point-source.

While the noise characteristics of the circuitry were not determined across all frequencies, a high SNR was found in the tested frequency domains ( $30 \mathrm{~dB}$ or better). The noise may depend on factors that were not controlled, eg, circuitry position relative to the MR scanner, and the performance may vary. Noise radiated by the circuitry was not a focus, as the SNR was found adequate for all current use cases.

For imaging of the emulated point-source, the slice selection and refocusing gradient waveforms were turned off, as the pick-up coil was found partially sensitive to $B_{1}(t)$. In cases where concurrent $B_{1}(t)$ and $B_{G}(t)$ is essential, the pick-up coil can be enclosed in Faraday shielding that only allows low frequency $B_{G}(t)$ fields to pass. The large difference in frequency between $B_{G}(t)$ and $B_{1}(t)$ makes the design of shielding relatively uncomplicated.

While $S_{\mathrm{e}}(t)$ and $S_{\mathrm{s}}(t)$ were chosen to only have $T_{2}$ relaxation appropriate for a true point-source, $S_{\mathrm{p}}(t)$ showed a faster $T_{2}^{*}$ signal decay, indicating that the physical phantom deviates from a true point-source, which also is supported by the slow oscillations seen in its phase evolution of $S_{\mathrm{p}}(t)$. 

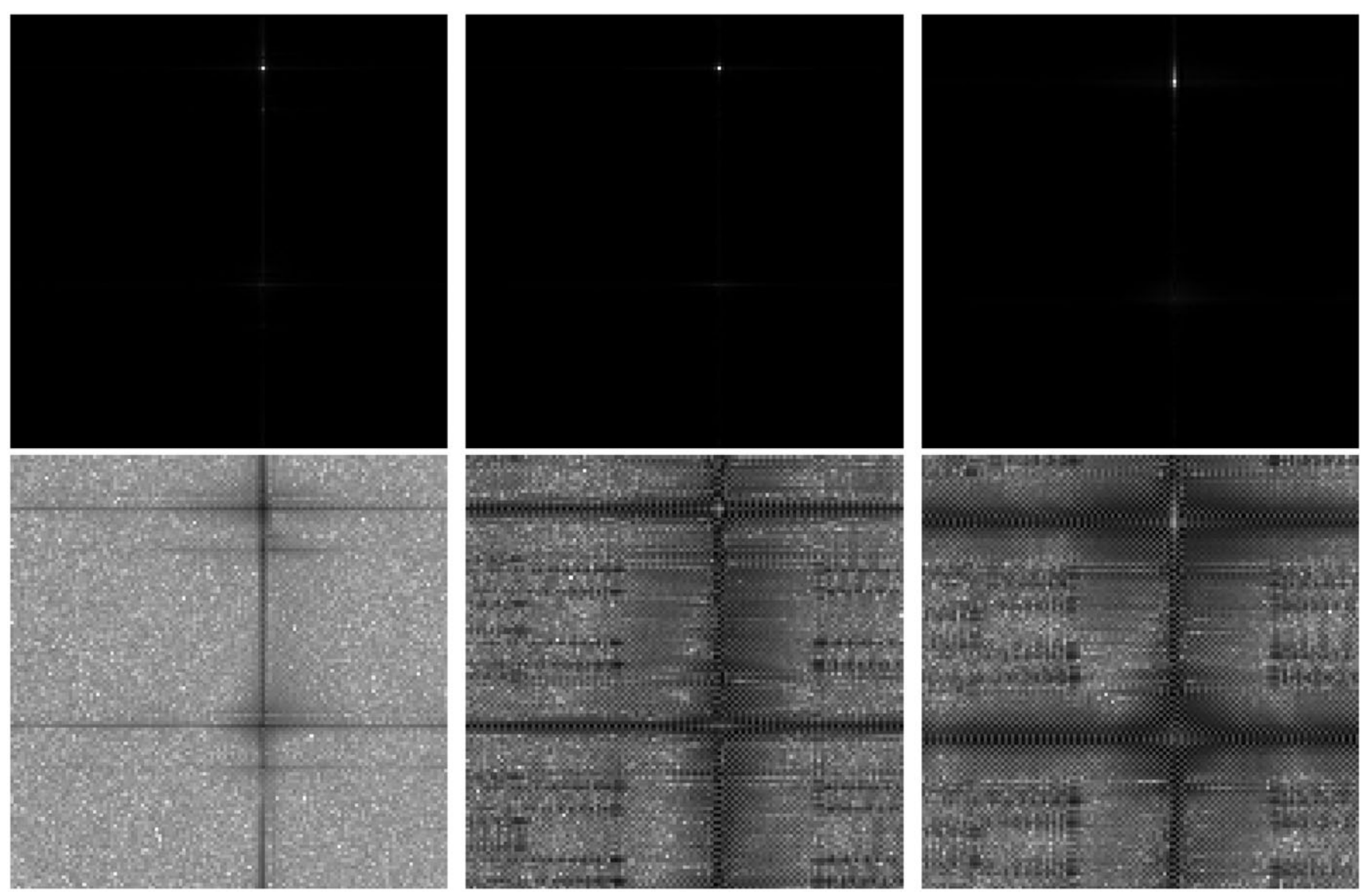

F IG URE 6 Reconstructed images of $S_{\mathrm{p}}(t)$ (left), $S_{\mathrm{e}}^{17}(t)$ (center), and $S_{\mathrm{e}}^{27}(t)$ (right), with linear (top) and logarithmic (bottom) scaling. The frequency encoding direction is left-right

A likely cause of this deviation is field inhomogeneities caused by the sample and its holder.

For fast imaging sequences, the latency introduced by passing a signal through the circuitry $(15 \mu \mathrm{s})$ amounts to multiple dwell-times. For SOIs where this latency is not negligible, it can be counteracted by delaying the scanner's signal acquisition accordingly, as it was done here for imaging of the emulated point-source. This can pose a challenge for concurrent MR and non-MR acquisition, as the delay should be accounted for in the reconstruction of the MR signal. As the delay is well-defined, this is relatively straight forward, either by using the Fourier shift theorem or updating the $k$ space grid used in the reconstruction. As the delay is dominated by digitization and communication from the ADC to the FPGA $(8 \mu \mathrm{s})$ and communication from the FPGA to the DDS subcircuitry $(4.5 \mu \mathrm{s})$, it can be significantly reduced by implementing parallel communication between sub-circuitries, and increasing the clock frequency of the circuitry. While implementation of both alterations would require extensive redesigning of the circuitry, they can potentially yield a delay shorter than or comparable to typical scanner dwell-times.

Inductively determining $B_{G}(t)$ during a single-shot EPI sequence is challenged by the large dynamic range of induced electromotive forces as it leads to increased digitization errors. ${ }^{19}$ EPI is additionally challenged by its relatively long readout-time, due to the intrinsic integration causing noise effects to accumulate over time. A higher precision is therefore expected for sequences generating electromotive forces of smaller dynamic range and with shorter readouttimes, as also evident from the highly decreased RMS error observed when effectively integrating over shorter time periods. Other methods for determining $B_{G}(t)$ may also improve the precision. Examples of such are field probes ${ }^{4,14,20}$ and Hall effect sensors ${ }^{21}$ which previously have been used in MRI, and yield a direct measure of the magnetic field, so integration is avoided. For showcasing the use of the presented circuitry, the precision obtained from inductive measures was, however, found adequate, and compared to other methods, inductive coils have the clear advantages of being easy to manufacture and use.

Electronic point-phantoms are potentially applicable for quantitative MRI, where an electronic phantom can provide reference signals for determining absolute concentrations. ${ }^{13}$ Another possible application is for motion tracking, by attaching one or multiple pick-up coils to a subject, and emulating individual point-sources. ${ }^{22}$ This could facilitate robust image registration, particularly relevant for low SNR images, 
such as diffusion weighted imaging, and low gamma MRI, eg, ${ }^{13} \mathrm{C}$ and ${ }^{23} \mathrm{Na}$, where this is a well-known challenge.

Besides emulating electronic phantoms, the presented circuitry also enables sampling of other SOIs. Since the position in $k$-space is proportional to the temporal integral of $f_{G}(t)$, the $k$-space trajectory can be determined with limited changes to the current experimental setup. While similar measures likely can be achieved with higher sensitivity using, eg, field probes, inductive measures are independent of signal relaxation, which simplifies their use significantly, and can be used independently of field inhomogeneity and of the nuclei targeted by the sequence. The applied gradient can be measured locally using, eg, a gradiometer ${ }^{19}$ or be globally estimated from the measured signal under a spatial linearity assumption and exploiting that the field is constant in the isocenter.

The RF power correlate measured by the circuitry (RF$\mathrm{Rx}$ ) does not yield phase information when measuring $B_{1}(t)$, which is a limitation, especially for multi-shot sequences. Demodulating the acquired $B_{1}(t)$ signal with the reference frequency of the MR scanner would allow sampling by LF-Rx, yielding both phase and amplitude measures. In order for the demodulated signal to have correct phase, clock synchronization between the MR scanner and the clock driving the deconvolution is necessary. It is possible that trigger pulses generated by the MR scanner can facilitate such clock synchronization, though the temporal precision might be confounding. Alternatively, the same crystal may be used for demodulation and modulation to ensure phase synchronization. With knowledge of the phase of $B_{1}(t)$ the signal from specific coherence pathways can, for example, be emulated, which is not directly feasible by imaging of a physical phantom.

In conclusion, the presented versatile, open-source circuitry and an MR scanner were used for sampling of nonMR signals, which provided a simple approach for acquiring signals of interest in synchrony with MR data acquisition. The circuitry was proven capable of performing real-time signal processing of multiple inputs to generate and transmit a single signal receivable by an MR scanner. Based on $B_{1}(t)$ power correlate measures and inductive $B_{G}(t)$ measures, a point-source signal was generated electronically during an EPI sequence and sampled by the scanner. The circuitry offers great flexibility, and facilitates sampling of most signals of interest during MR acquisition, and is a useful and inexpensive tool for, eg, scanner monitoring and methods development.

\section{ORCID}

Jan Ole Pedersen (iD http://orcid.org/0000-0002-3099-2106 Lars G. Hanson (iD http://orcid.org/0000-0002-8204-6912

\section{REFERENCES}

1. Gotman J, Kobayashi E, Bagshaw AP, Bénar CG, Dubeau F. Combining EEG and fMRI: a multimodal tool for epilepsy research. J Magn Reson Imaging. 2006;23(6):906-920.

2. Huster RJ, Debener S, Eichele T, Herrmann CS. Methods for simultaneous EEG-fMRI: an introductory review. J Neurosci. 2012;32(18):6053-6060.

3. Duyn JH, Yang Y, Frank JA, van der Veen JW. Simple correction method for k-space trajectory deviations in MRI. J Magn Reson. 1998;132(1):150-153.

4. Kasper L, Bollmann S, Vannesjo SJ, et al. Monitoring, analysis, and correction of magnetic field fluctuations in echo planar imaging time series. Magn Reson Med. 2015;74(2):396-409.

5. Mason GF, Harshbarger T, Hetherington HP, Zhang Y, Pohost GM, Twieg DB. A method to measure arbitrary k-space trajectories for rapid MR imaging. Magn Reson Med. 1997;38(3):492496.

6. Bresch E, Nielsen J, Nayak K, Narayanan S. Synchronized and noise-robust audio recordings during realtime magnetic resonance imaging scans. J Acoust Soc Am. 2006;120(4):1791-1794.

7. Goldman RI, Stern JM, Engel J, Cohen MS. Acquiring simultaneous EEG and functional MRI. Clin Neurophysiol. 2000;111:19741980.

8. Anami K, Mori T, Tanaka F, et al. Stepping stone sampling for retrieving artifact-free electroencephalogram during functional magnetic resonance imaging. NeuroImage. 2003;19(2):281-295.

9. Hanson LG, Hanson CG. A multi-frequency amplitude modulator for encoding electrical signals in MR images. In: Proceedings 14th Scientific Meeting, International Society for Magnetic Resonance in Medicine Seattle; 2006:2388.

10. Hanson LG, Lund TE, Hanson CG. Encoding of electrophysiology and other signals in MR images. J Magn Reson Imaging. 2007;25(5):1059-1066.

11. Navest RJM, Andreychenko A, Lagendijk JJW, van den Berg CAT. Prospective respiration detection in magnetic resonance imaging by a non-interfering noise navigator. IEEE Trans Med Imaging. 2018;62:1-10.

12. Schroeder L, Wetzl J, Maier A, et al. A Novel method for contact-free cardiac synchronization using the pilot tone navigator. Proc Intl Soc Magn Reson Med. 2016;24:410.

13. Salvati R, Hitti E, Bellanger JJ, Saint-Jalmes H, Gambarota G. Fat ViP MRI: virtual phantom magnetic resonance imaging of water-fat systems. Magn Reson Imaging. 2016;34(5):617-623.

14. Dietrich BE, Brunner DO, Wilm BJ, et al. A field camera for MR sequence monitoring and system analysis. Magn Reson Med. 2016;75(4):1831-1840.

15. Haacke EM, Brown RW, Thompson MR, Venkatesan R. Magnetic Resonance Imaging: Physical Principles and Sequence Design. Hoboken, NJ: Wiley; 1999.

16. Bernstein MA, King KF, Zhou XJ. Handbook of MRI Pulse Sequences. Cambridge, MA: Academic Press; 2004.

17. Zur Y, Stokar S. An algorithm for eddy currents symmetrization and compensation. Magn Reson Med. 1996;35(2):252-260.

18. Greengard L, Lee J-Y. Accelerating the nonuniform fast Fourier transform. SIAM Rev. 2004;46(3):443-454.

19. Senaj V, Guillot G, Darrasse L. Inductive measurement of magnetic field gradients for magnetic resonance imaging. Rev Sci Instrum. 1998;69(6):2400-2405. 
20. Vannesjo SJ, Haeberlin M, Kasper L, et al. Gradient system characterization by impulse response measurements with a dynamic field camera. Magn Reson Med. 2013;69(2):583-593.

21. Schell J, Kammerer J, Hébrard L, Breton E, Gounot D, Cuvillon L. Towards a Hall effect magnetic tracking device for MRI. 35th Annual International Conference of the IEEE EMBS; 2013:29642967.

22. Ooi MB, Krueger S, Thomas WJ, Swaminathan SV, Brown TR. Prospective real-time correction for arbitrary head motion using active markers. Magn Reson Med. 2009;62(4):943-954.

How to cite this article: Pedersen JO, Hanson CG, Xue R, Hanson LG. General purpose electronics for real-time processing and encoding of non-MR data in MR acquisitions. Concepts Magn Reson Part B.

2018;e21385. https://doi.org/10.1002/cmr.b.21385 